\title{
Towards a Miniaturized Photon Counting Laser Altimeter and Stereoscopic Camera Instrument Suite for Microsatellites
}

\author{
S.G. Moon ${ }^{1}$, S. Hannemann, M. Collon. cosine Research B.V., Niels Bohrweg 11, 2333 CA \\ Leiden, The Netherland
}

K. Wielinga, E. Kroesbergen. Mecon Engineering B.V., Koopmanslaan 25, 7005 BK

Doetinchem, The Netherlands

\begin{abstract}
J. Harris. Swiss Space Technology, Route de Chavalet 2, 18349 Champery, Switzerland
E. Gill, D. Maessen. Delft University of Technology, Kluyverweg 1, $2629 \mathrm{HS}$ Delft

The Netherlands
\end{abstract}

IAA-B7-0310P

\begin{abstract}
In the following we review the optimization for microsatellite deployment of a highly integrated payload suite comprising a high resolution camera, an additional camera for stereoscopic imaging, and a single photon counting laser altimeter. This payload suite, the 'Stereo Imaging Laser Altimeter' SILAT has been designated for deployment aboard the FAST microsatellite formation mission for Earth observation. This instrument suite has been designed for a Jupiter mission, but has been redesigned and optimized for an Earth observation mission. This paper reviews the simulated Earth observation performance, the design modifications made for the mission and the optimization of the design for microsatellite use. Mass and power budgets are used to demonstrate the changes and the performance analysis is represented trough the simulation results. It is expected that the optimization will reduce the mass of the instrument by approximately $201 \%$ without compromising the performance of the instrument. In addition, results from breadboarding experiments of individual instrument components will be presented to show the progress from design optimization towards the FAST flight model.
\end{abstract}

\section{Introduction}

Understanding global climate change is a task that could be well suited to microsatellites, which can be efficiently designed to uniquely address key issues and specific scientific questions. A collaborative mission consisting of two spacecraft has been proposed jointly by TU Delft (The Netherlands) and the University of Tsinghua (China) for launch in 2011. This mission will be dedicated to the investigation of climate change by examining, among other objectives, atmospheric aerosol content and surface topography. Both spacecraft will carry a spectropolarimeter, which measures the intensity and polarisation of reflected and scattered radiation as a function of wavelength. In addition, each will also carry a topographic imager: one imager will be a radar, and the other a laser altimeter.

Discussed here is the design and development of SILAT, a Stereo Imaging Laser AlTimeter, which could satisfy the requirements of such a mission. This instrument combines a high resolution nadir-pointing camera, a low resolution offset stereo camera, and a single photon counting laser altimeter. The laser altimeter is characterized by a low power, high repetition rate design. SILAT features a highly integrated optical design and shared data processing unit, enabling a compact and low-mass package. In addition, the single photon counting mode of operation uses considerably less power than traditional laser altimeters. In addition to the instrument itself, factors involved with accommodating SILAT on a

${ }^{1}$ Corresponding Author. Tel: +31 71524 1843, Fax:+31715284963 email: s.moon@cosine.nl

$7^{\text {th }}$ IAA Symposium on Small Satellites for Earth Observation, May 4-8, 2009 
microsatellite (referred to as the HIPS microsatellite in the following) will also be addressed. This includes configuration of the instrument, integration with the microsatellite bus, and the resource requirements imposed on the satellite by SILAT. The microsatellite bus has been designed on the basis of a Europe-oriented Earth observation mission.

\section{Mission Overview}

\subsection{The FAST Mission}

In early 2008 the University of Technology Delft in the Netherlands and the University of Tsinghua in China proposed a collaborative formation flying mission consisting of two microsatellites. These spacecraft would each be equipped with two main payloads. The first is a spectropolarimeter, which is used to measure the intensity and polarisation of reflected and scattered radiation in the Earth's atmosphere as a function of wavelength. The second payload is a topographic imager; and, in the interest of collecting as much distinct data for a target location, each microsatellite will be carrying a distinct type of topographic imager. One microsatellite will carry a radar instrument and the other will carry a laser altimeter. In the case of the FAST-D satellite, SILAT will fulfil the laser altimeter role. SILAT is an ideal payload as it can provide not only a photon counting laser altimeter, but also a high resolution visible spectrum camera and a single band stereo camera. This instrument suite is heavily integrated and optimized, which reduces the mass, volume and power requirements, making it ideal for deployment on a microsatellite in the $25 \mathrm{~kg}$ to $50 \mathrm{~kg}$ range. The SPEX instrument will fly as a second payload on the FAST mission to collect data on aerosol particles in the atmosphere. In addition to providing scientific data for the FAST mission, the use of the SILAT payload would act as an in situ validation of the single photon counting laser altimeter concept.

\section{Scientific Objectives}

SILAT is capable of delivering varied scientific data, owing to its modular design and removable instruments. The mission parameters were formulated based upon a mission to provide regular high resolution maps of Europe in general and the Netherlands in particular. In addition, stereoscopic images and height information for incorporation into digital elevation models (DEM)s will be provided by SILAT. This aspect of the mission will be able to provide high resolution height and spectroscopic detail of the Dutch dykes and dunes with relatively high frequency. In conjunction, SPEX can provide information about atmospheric particles, which in combination can result in valuable data for monitoring and predicting the threats to sensitive areas of the Netherlands.

The FAST mission, which will feature two microsatellites, has science objectives that include, but are not limited to the above. The two satellites will monitor fixed and liquid particles present in the atmosphere, such as soot particles. The two satellites can also generate stereoscopic images, and altimetry measurements of snow and ice levels on earth. The goal of the formation flying is to provide new, valuable scientific data in the fields of air quality and climate change while utilizing novel satellite concepts; namely, microsatellite platforms and formation flying. Doing so explores the benefits of alternatives to large, single satellite Earth observation missions.

\section{Description of SILAT Integrated Payload Suite}

SILAT is an highly integrated payload that is composed of three separate instruments. SILAT is the product of design studies conducted at cosine for the purpose of developing the concept of the use of highly integrated payload suites for future planetary exploration

$7^{\text {th }}$ IAA Symposium on Small Satellites for Earth Observation, May 4-8, 2009 
missions [Kraft, 2005]. These studies have evaluated the SILAT design at both Mercury and Europa. This section will describe each component of SILAT and detail the science objectives achievable, as well as the requirements, for each component.

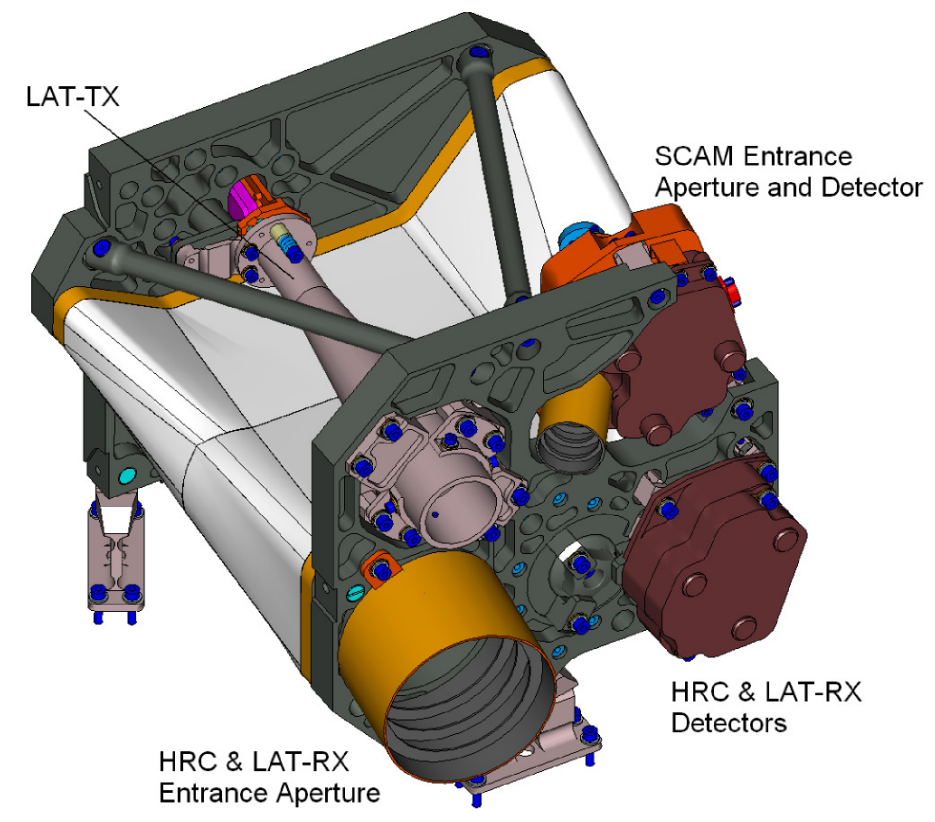

Figure 1: SILAT integrated payload suite with sub-instruments identified

\subsection{SILAT Features}

SILAT utilizes a number of unique features that allow it to be used in a variety of planetary theatres. Thermally, SILAT is as isolated as possible from the spacecraft temperature gradient: radiatively through the use of multi-layer insulation (MLI) on the exterior of the instrument suite, and conductively via the mounting legs, which act as a thermal choke. Furthermore, the body of SILAT is, as much as possible, composed of silicon carbide (SiC). This material has a thermal conductivity of $180 \mathrm{~W} /(\mathrm{m} \cdot \mathrm{K}$ ) (on par with that of 6061-T aluminium) but its Coefficient of Thermal Expansion (CTE) an order of magnitude lower $\left(2.2 \times 10^{-6} \mathrm{~K}^{-1}\right.$ against $\left.23.9 \times 10^{-6} \mathrm{~K}^{-1}\right)$. This promotes the elimination of thermal gradients in the instrument suite, thus inducing uniform expansion. This renders SILAT resistant to misalignment from thermal expansion.

SILAT also features custom fasteners for the optical components. These fasteners allow the optical components to be minutely adjusted, to the micrometer level. They are made from $\mathrm{SiC}$ as well, keeping in line with the SILAT design philosophy.

Another facet of SILAT is that it employs passive elements as much as possible. While the LAT is an obvious exception, SILAT contains no moving parts, and all cooling devices are passive; only cold fingers and radiators are used. This increases the reliability of the payload suite, which is essential for deep space missions.

\subsection{Earth Orbit Optimization -Radiation Shielding}

The materials, and material thicknesses, specified in the original SILAT design were based on an evaluation of the radiation environment in an orbit around Europa at Jupiter. A Low Earth Orbit (LEO), even one close to the inner Van Allen radiation belt, experiences a significantly less harsh flux of harmful radiation. As such, the radiation shielding of the instrument suite has to be re-evaluated. This includes both the materials used, their thickness and their orientation if there is a directional dependency. 
Densimet, a tungsten alloy, is an excellent radiation shield, but is very dense. Its use on the Jupiter configuration as a shield for the electronics was driven by the desire to keep the envelope of SILAT as small as possible. Given the reduced radiation environment, aluminium can provide the same respective shielding while maintaining the volume profile of the instrument. As such, the use of Densimet is not necessary for the FAST configuration, and is replaced by aluminium.

Since the radiation environment is less harsh, the thickness of the SiC baseplate can be reduced as well. Based on the evaluation of the radiation environment, the thickness can be reduced by approximately $50 \%$ saving approximately $1 \mathrm{~kg}$ of mass.

The new structural dimensions of the redesigned SILAT will be presented at the 7th IAA Conference on Small Satellites.

\section{Performance of SILAT for Earth Observation}

\subsection{Orbital parameters}

Based on the scientific goals described in $\S 3$, a sun synchronous orbit at $700 \mathrm{~km}$ has been chosen for the microsatellite. The details of the orbital parameters are summarized in Table 1.

Table 1: Parameters of Sun Synchronous Orbit

\begin{tabular}{|c|c|}
\hline Type & SSO \\
\hline Inclination & $98.7^{\circ}$ \\
\hline Altitude & $700 \mathrm{~km}$ \\
\hline Velocity & $7.51 \mathrm{~km} / \mathrm{s}$ \\
\hline Orbital Period & $98.6 \mathrm{~min}$ \\
\hline Sunlit Time & $61.1 \mathrm{~min}$ \\
\hline Eclipse Time & $37.5 \mathrm{~min}$ \\
\hline Sunlit Fraction & 0.62 \\
\hline
\end{tabular}

This orbit has been chosen based on a trade-off between the resolution, swath width and revisit of the SILAT payload with respect to the mapping of Europe. $700 \mathrm{~km}$ established a reasonable spatial resolution of the HRC of $21.9 \mathrm{~m}$ ( Table 2) while allowing $100 \%$ coverage of Europe to be achieved in 100 days, and the Netherlands in 52 days. The sunlit fraction of the orbit is a parameter used to gauge the amount of power available from solar irradiance, and drives the solar array design.

This orbit is also beneficial in that it is launched to relatively frequently. This makes it a convenient selection for a piggy-back launch strategy, which is the preferred for a microsatellite launch.

\subsection{Instrument performance}

In order to quantify the performance of SILAT for Earth observation, simulations have been carried out at cosine. For this purpose, MathCAD sheets have been developed to calculate performance parameters for the HRC and LAT. The program uses orbital parameters described in $\S 5.1$ as the mission input. It calculates the radiometric level of the HRC and LAT detectors, based on parameters such as the Sun-Earth distance, the Earth-satellite distance, the albedo of different surfaces, the atmospheric transmittance, the aperture size and overall transmission efficiency of the imaging optics and filters. From the detector 
specifications (dark count noise, read-out noise, quantum efficiency, pixel size and dead time) the simulations calculates the SNR.

In addition the program takes the basic characteristics of the laser into account. Thus, the performance calculations of LAT are based on parameters such as repetition rate, pulse energy, wavelength and pulse duration. For both the HRC and LAT the simulation has additional variables to reflect various illumination conditions such as cloud, clear sky, day, night.

\subsection{HRC performance}

Based on the orbital parameters given in Table 1 and the CCD detector characteristics described in [Moon, 2008] the performance parameters for the HRC, presented in Table 2, are calculated. These parameters are fed into an Earth observation simulation code (or in the case of non-Earth planetary exploration, to the HIPSIM simulator), and the SNR is obtained.

Table 2: Orbit dependant HRC characteristics

\begin{tabular}{|c|c|}
\hline Ground Velocity & $6.77 \mathrm{~km} / \mathrm{s}$ \\
\hline Focal Distance & $320 \mathrm{~mm}$ \\
\hline F\# & 4.9 \\
\hline IFOV & $31.5 \mu \mathrm{rad}$ \\
\hline Single Pixel Footprint & $21.9 \mathrm{~m}$ \\
\hline Integration Time & $1.62 \mathrm{~ms}$ \\
\hline FOV & $3.67^{\circ}$ \\
\hline Swath Width & $44.8 \mathrm{~km}$ \\
\hline
\end{tabular}

The integration time is defined as the time the spacecraft requires to travel across half a pixel footprint. Although the resolution of the HRC does not compete with larger, more powerful optical payloads, it will be sufficient to carry out the environmental surveys described by the HIPS microsatellite mission and the FAST science objectives.

From the radiometric level and the performance characteristics on the HRC detector the signal to noise ratio was calculated for all three wavelength bands corresponding to the filter attached to the HRC detector. The result is shown in Figure 2, where the SNR is depicted as a function of the orbital argument. The maximum SNR for one orbit exceeds 200 in all three wavelength bands. The green band even reaches values of 500. However, for historic reasons, atmospheric effects are not fully taken into account yet. While transmission losses through the atmosphere have been taken into account, the simulation that generates the SNR does currently not reflect the increased background light levels caused by atmospheric Rayleigh scattering. Work has begun on including this in the simulation model, however the additional module is not complete and results have not yet been generated. 


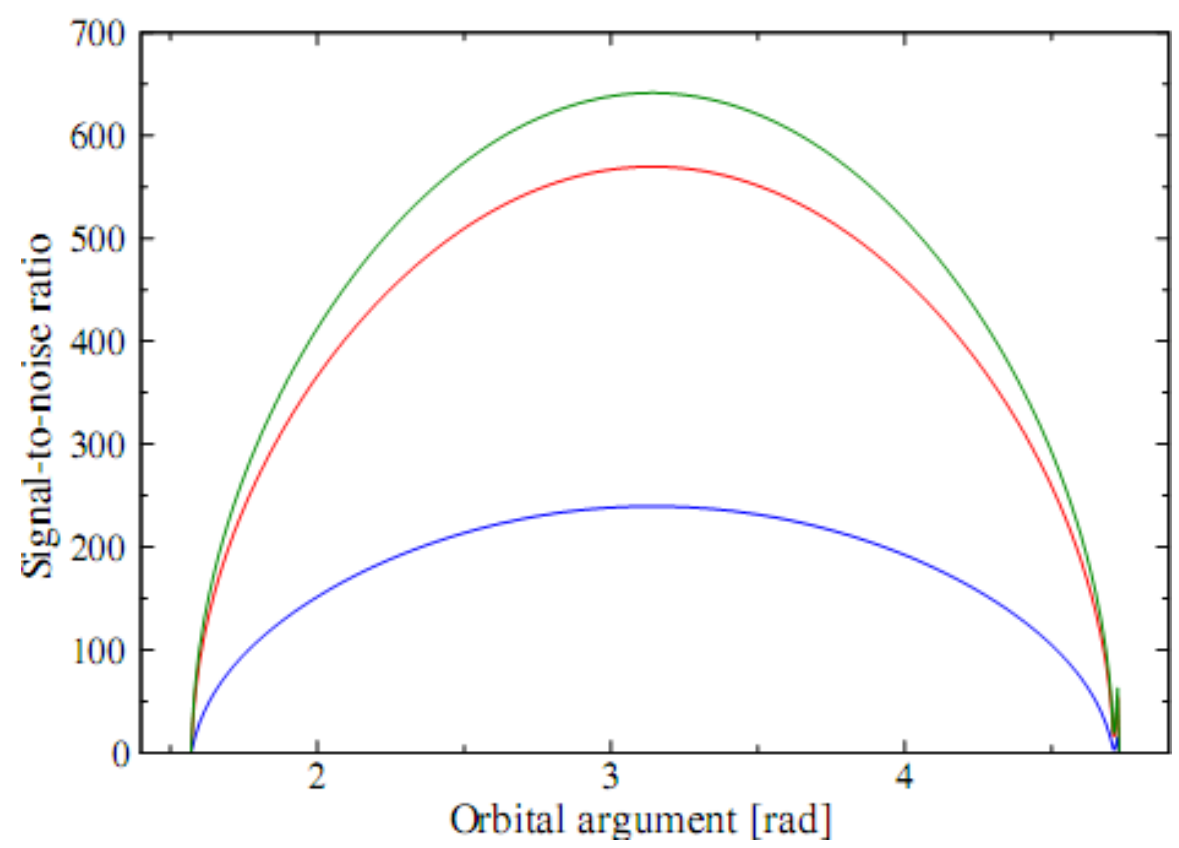

Figure 2: Plot of Signal-to-Noise vs. orbital argument for an SSO SILAT. Note that the argument at which the SNR falls to zero are the entry/exit points from eclipse.

\subsection{SCAM Performance}

Due to the limitations of the poster session, the performance of the SCAM instrument has been omitted. The Earth Observation performance of this instrument on SILAT is covered in Moon, 2008.

\subsection{LAT performance}

The performance of the LAT can be characterized at different levels. Since the instrument is based on time-of-flight measurement, the ultimate limit of the achievable height resolution is correlated with the pulse duration of the laser in use. Light travels $30 \mathrm{~cm}$ in $1 \mathrm{~ns}$. Since a photon emitted by the LAT-TX will travel the distance from the instrument to the Earth's surface twice during its flight, a height difference of $15 \mathrm{~cm}$ will result in a path length difference of $30 \mathrm{~cm}$, which will be detectable by the detector if it operates at a speed of $1 \mathrm{~ns}$. Thus the ultimate height resolution is about $15 \mathrm{~cm}$. With good statistics, that value can be improved by the familiar $1 / \sqrt{ } N_{\text {sh }}$ factor, where $N_{\text {sh }}$ represents the number of shots taken. Parameters for the LAT performance are shown in Table 3.

Table 3. Performance parameters of the laser altimeter

\begin{tabular}{|c|c|}
\hline Ground Velocity & $6.77 \mathrm{~km} / \mathrm{s}$ \\
\hline Detector Size & $20 \mu \mathrm{m}$ \\
\hline IFOV-RX & $63 \mu \mathrm{rad}$ \\
\hline Footprint Size (RX) & $43.8 \mathrm{~m}$ \\
\hline Footprint Size (TX) & $17 \mathrm{~m}$ \\
\hline Repetition Rate & $10 \mathrm{kHz}$ \\
\hline \#-pulses/footprint & 25 \\
\hline Time-of-Flight & $4.67 \mathrm{~ms}$ \\
\hline
\end{tabular}

The next important set of parameters consists of the ground speed of the spacecraft, the repetition rate of the laser, and the footprint sizes of the transmitter and receiver optics. These parameters determine the statistics with which the ground track will be scanned. Within the time the spacecraft passes a given distance a number of shots will be emitted where mutual overlap of the individual footprints of the laser pulses will provide the 
redundancy for improving the statistics. With the current parameters $(10 \mathrm{kHz}$ repetition rate, $700 \mathrm{~km}$ altitude) about 25 pulses will be emitted during the time the spacecraft passes the distance equivalent to one LAT-TX footprint size.

From the height of the spacecraft, the atmospheric transmission, the transmitter and receiver properties and the laser characteristics it is possible to calculate the number of detected photons per second (Altimeter equation, [Degnan, 2002]. Preliminary results indicate that the that rate is approximately 25 detected photons per second. More detailed investigations on how that rate can be increased on the HIPS microsatellite orbit are still underway.

As stated earlier, the CRR converts the returned pulses to a resolution of the height based on statistical distributions of the TOF of the returned photons, thus this factor contributes directly to the spatial resolution achievable by the LAT.

It is also of crucial importance that the orbital height corresponds to a TOF of the LAT signal photons of slightly less than $5 \mathrm{~ms}$. Within that time, the spacecraft pointing must be stable within a fraction of the instantaneous field-of-view (IFOV) of the LAT-RX. Otherwise the LAT will lose signal and fail to register any of the returning photons.

\section{Earth Orbit Optimization - Breadboarding Results}

cosine has recently presented a successful demonstration of the photon-counting laser altimeter breadboard. This breadboard is capable of controlling a pulse emitted from a prototype microchip laser, detecting said pulse and then sorting and analyzing the resulting data to form a distance profile. The demonstration so far have been limited to distance measurements of objects in various environments within labs, indoors and outdoors in fair weather, with an effective range of $100 \mathrm{~m}$. Preliminary demonstrations for external parties have been given, but the components have recently undergone fine tuning and additional development in order to increase the performance of the breadboard.

The current status of the laser altimeter is within the integration stage. The full breadboard including emitter, receiver, control electronics and data-processing FPGA will be assembled during the first week of May, with the full field testing conducted during May in Leiden, Netherlands. The instrument is expected to exit the breadboard stage during May and enter full prototype status on an engineering model in the summer.

The current goal of this project is to produce two pre-flight models, one of SILAT, and one of the MPS, for a micro- and nano-satellite launch opportunity respectively, in partnership with the FAST mission with TU Delft and the Delfi-NexT mission, also with TU Delft.

In addition to the breadboarding, SILAT is also being optimized for the FAST project by optimizing the design for a LEO mission. This includes a detailed look at the radiation environment in the proposed FAST orbit, and a reduction of the radiation shielding materials and a change in the shielding strategy, while maintaining the desired performance. A parametric study, which employs a comparative analysis of previous SILAT operations for Jupiter against the LEO radiation environment, has resulted in a new mass of $5.2 \mathrm{~kg}$ and the replace of very dense, very radiation hard shielding materials with aluminium or stainless steel. Previous studies have shown that the thermal and control electronics subsystems will be appropriate for a LEO application of SILAT. With this knowledge, and the knowledge of the behaviour of the optical systems with respect to Earth's atmosphere, SILAT is ready to move into a full, integrated engineering model.

In the context of the FAST mission, there is approximately 1 year to develop and test an engineering model of SILAT, followed by a 6 month period to produce the FAST-ready flight model. cosine and the University of Technology in Delft are working closely to keep the satellite and payload development on schedule.

$7^{\text {th }}$ IAA Symposium on Small Satellites for Earth Observation, May 4-8, 2009 


\section{Other Micropayloads}

In addition to developing the laser altimeter, cosine has developed two other micropayloads using the same development methodology as SILAT. These two micropayloads are the Highly Integrated Broadband HyperSpectral Imager and Spectrometer (HIBRIS) and the Multifunctional Particle Spectrometer. As these are represented on the symposium poster, they are described briefly below.

\subsection{HIBRIS}

HIBRIS has been developed in parallel with SILAT, using the same approach to development. Instruments have been integrated and miniaturized to provide maximum scientific value within a small volume, with low mass and power requirements. HIBRIS consists of a hyper-spectral Near Infrared (NIR) spectrometer combined with a low resolution Thermal Infrared (TIR) imager. The instrument provides wide area thermal imagers with detailed spectroscopy over the NIR wavelength range. Like SILAT, HIBRIS is meant as a payload module; it is connected as a fully independent unit to a small or large satellite. HIBRIS is thermally and vibrationally isolated, and requires less than $4.5 \mathrm{~W}$ of input power from the spacecraft. HIBRIS has a mass of approximately $7 \mathrm{~kg}$. HIBRIS is currently in the breadboard stage. A fully functional engineering model is planned for 2010.

\subsection{MPS}

The Multifunctional Particle Spectrometer is a variable role energetic particle detector. The MPS is only $6 \times 6 \times 8 \mathrm{~cm}$ large module, occupying a very small volume. The instrument has multiple configurations however, allowing it to fulfil a variety of roles from simple particle monitoring collecting counts of different particle types, to full-fledged particle analysis. Its full capabilities include determining the type, energy and angle of incidence of incoming electrons, protons, gamma rays and ionized particles. The MPS can operate at a rate on the order of $\mathrm{kHz}$.

The MPS is currently in the prototype phase. The control electronics breadboards have been completed, and the optical and semi-conductor are being prototyped. The short term goal is to complete an engineering model before Autumn 2009. The MPS currently has a launch opportunity on the TU Delft nano-satellite Delfi-NexT.

\section{Summary and Outlook}

SILAT is a highly integrated payload suite that contains three separate visible spectrum remote sensing instruments: a high resolution camera; a reduced resolution stereo camera and a photon-counting laser altimeter. SILAT has been designed for use in exploration missions to Mercury and Europa, and this paper has evaluated the feasibility of using such a payload for Earth observation such as the upcoming FAST mission at the Technical University of Delft. Contained within this paper has been an evaluation of the performance of the SILAT integrated payload suite for an Earth observation mission, and a preliminary investigation into the microsatellite support platform that SILAT would require. Performance calculations have been done by cosine, and the microsatellite design by ISIS. The MathCAD simulations indicate that the HRC and SCAM will have high SNR ratios. Though the full impact of atmospheric effects must still be modelled, it is not expected that this will appreciably reduce the utility of SILAT in an Earth observation role. The resolution of these two instruments will also be increased if used in the upcoming FAST mission, as FAST is proposed to fly $50 \mathrm{~km}$ lower than the orbit indicated in this paper. 


\section{References}

M. Bentley, F. Varlet and S. Kraft. 2007. Consolidation of HIPS FM Preliminary Design. cosine internal communication [CR-HDD-TN-01, Issue 02]. June 06, 2007

M. Collon et al. 2005. HIPS Breadboard Engineering Plan. Cosine internal document [CRHIPS-PL-EP, Issue 01]. September 8, 2005

A.J. Court et al.(TNO). 2007. TNO-SPEX (Spectropolarimeter for Planetary Exploration). Available at http://www.tno.nl, accessed May 1, 2009.\}

J.J. Degnan. 2002. Photon-counting multikilohertz altimeters for airborne and spaceborne topographic measurements. Journal of Geodynamics. Vol. 35,pp 503-549

S. Moon et al. A Miniaturized Laser Altimeter and Stereo Camera For A Microsatellite Formation Mission. In ESA Small Satellites Systems and Services - The 4S Symposium. Rhodes, Greece, 26-30 May 2008. ESA:Netherlands 